\title{
Virtual Reality: Development of an Integrated Learning Environment for Education
}

\author{
Ahmed Al-Gindy, Chema Felix, Ali Ahmed, Amani Matoug, and Munia Alkhidir
}

\begin{abstract}
The new paradigm shift of education where learners should learn real-life scenarios and solve real-life problems has created a major challenge. Students are passive and detached and may struggle to see the relevance of what they are learning to their lives. The recent growth of emerging technologies has launched terms such as virtual reality (VR), augmented reality (AR) and mixed reality (MR) into mainstream topic. Virtual reality, an integrated, hands-on tool for learning, can play a unique role in addressing these educational challenges. The proposed research demonstrates using the knowledge of integrated disciplines with virtual reality. COSPACES and MERGE cube add-on have been used to develop a simulation platform to teach water cycle process for young kids. This type of integration develops learners' $21 \mathrm{st}$ century skills to be decision makers, problem solvers, lifelong learners, creators, innovators, and to think critically in solving problems.
\end{abstract}

Index Terms-Multimedia, augmented reality, virtual reality, integrated learning.

\section{INTRODUCTION}

In the age of technological and $4^{\text {th }}$ industrial advancements educators believe that it is time to change mindsets and bring in new concepts in learning that will drive innovation across the board. This will transform education from a typical teacher-centric classroom to a child-centred one by encouraging a curriculum that is driven by problem-solving discovery and experimental learning.

With the growth of smartphones and digital devices, many ideas have been established to go together with the latest innovations. One such concept is virtual reality (VR), which allows users to integrate themselves in a computer-based generated environment. The industry of emerging technologies has launched the definitions of virtual reality (VR), augmented reality (AR) and mixed reality (MR) into mainstream discourse. Briefly, virtual reality integrates users in a fully simulated digital atmosphere; augmented reality (AR) overlaps virtual objects on the real-world environment; and, mixed reality (MR) integrate virtual objects on to the real world and often allows users to interact with these objects [1]. Virtual reality has been everywhere for some years and includes miscellaneous applications from accurate training simulations used by NASA to multi-entertainer 3D virtual worlds such as Second Life. There is no accepted definition of integrated virtual reality; however, it is usually classified as a type of VR through a head mounted display" (HMD).

Manuscript received October 12, 2019; revised January 18, 2020.

The authors are with Department of Electrical Engineering, Canadian University Dubai, UAE (e-mail: agindy@cud.ac.ae).
HMD is a headset that offers visual effects and multimedia directly to the eyes so that wherever a user looks the display is in front of the eyes which pathways (in various tracks and degrees) the user's location in space. This technology forms a feeling of presence or 'being there' in the virtual world or in the case of a networked computer environment, co-presence or 'a sense of being there with others [2]. For feelings of presence to happen two factors are vital. These are place illusion, which is a strong feeling that you are actually in the virtual place, and believability illusion, which is a prevailing feeling that what is happening in the virtual place is occurring [3].

For some years, VR learning affordances (properties that can allow for learning) have been documented [4] with some suggesting that the technology has the potential to completely transform education [5].

Authors in [6] argue that 3D virtual learning environments (3D VLEs) can enhance three-dimensional knowledge; ease experimental learning that would otherwise be impossible or unfeasible in the real world. VR can also progress transfer of knowledge and skills learned in virtual atmospheres to real situations. It may also increase enthusiasm and engagement in learning that leads to richer "collaborations.

Authors in [7] suggesting that 3D virtual learning environments can offer new openings for creativity in learning through role play and mentoring. This in turn leads to open learning spaces for practice and discovery, experimentation and user created content. The expanded skills for learner-led problem and inquiry-based learning. Given the recent commercial availability of VR, it is unsurprising that there is limited research on using VR in school classrooms [8].

There is a growing works on VR for learning in higher education, particularly in engineering, science and medicine and some of this may have consequences for using VR in schools. For example, [9] reviews the benefits of virtual laboratories in science, technology and engineering for higher education, highlighting the possible cost-effectiveness of high-quality virtual laboratories and the way where several students can access virtual equipment which is, unlike physical equipment resistant to damage. They also suggest that virtual laboratories can make the 'unseen' as the cover of equipment can be easily removed or made transparent to how the workings of the inner structure (in a robot, for example, it is possible to easily to reveal all its working parts). However [9] advise that virtual laboratories are usually time consuming to advance. In addition, students may not take fake experiences as seriously as real ones, and that in the final or advances stages of training and learning, there is no substitute for genuine hands-on experience with physical 
equipment. The results from a recent science laboratory experiment with university students, which compared learning in desktop VR with integrated (HMD) VR, suggested that while students felt a greater sense of presence in VR they may have experienced cognitive overload resulting in poorer test performance [10].

Some may argue that using VR for education is unnecessary since videos and shows are just effective at explaining content. However, VR offers a level of integration that is unparalleled by traditional mediums. With VR, users feel more connected to the content because they can personally, interact with and deploy it. [3] Compared with television, where the experience is from an outside point of view, VR offers a realistic first-person perspective. This key aspect gives VR large potential for educational uses.

Education is being reshaped for students and teachers alike through new, innovative teaching methods, advancements in technology, and programs designed to make that technology available to as many schools as possible. In the last five years, education has been changing in so many ways so quickly that it can be difficult to tell what all this upheaval will lead to. But in a broad sense, it is not hard to see that all these changes are pointing in one direction: increasing educational opportunities" for all students.

Class styles are changing; teachers are giving whole-class instruction where they deliver information to students. More classes are now online or blended, which is a mix of online and face-to-face. Classes are also being flipped, where teachers assign a video lecture for homework, and students use the class time to work collaboratively and apply the knowledge to various problems. Add in brain research that explains exactly how students learn and the data explosion that can tell teachers specifically which students understood her lesson before it's over.

\section{AR AND VR PLATFORMS FOR EDUCATION}

Traditional educational methods are slowly becoming supplanted by more advanced techniques. Educators constantly seek to develop new and exciting methods to make education more interactive and effective for the students. The education experience is being reshaped for students and teachers alike through new innovative teaching methods, advancements in technology, and programs designed to make technology more readily available. [7] In particular, VR in education helps students retain information better, as it creates a unique, individualized environment that cannot be replicated in the classroom setting.

Unity is a development platform that is used to create 2D and 3D games. Students can Create games, simulations and VR/AR tools that improve their skills, knowledge and performance. For Example, CAE Vimedix AR help students learn ultrasound by allowing them to visualize anatomy and ultrasound cut plane in real-time.

In Addition, Unity has many virtual solutions for real world applications such as, automotive, transportation and manufacturing, film transportation and cinemas, architecture, engineering and construction.

PG\&E Becker pilot service training is an interactive 3D application developed by unity. (PG\&E) is one of the largest natural gas and electric energy companies in the US, providing natural gas and electric service to 16 million people throughout a 70,000-square-mile service area in northern and central California. Founded: 1905, Location: HQ San Francisco, CA, Employee \#: 23,500, Revenue: \$17.6 Billion. Every 5 years, PG\&E gas service technicians are required to perform a complete tear-down and re-build of a GE Becker VRP-600-CH Pilot, as part of preventive maintenance. There are up to 20 pilots in a Field Service Representative's (FSR) territory. Due to the complex nature of parts and components, both the error rate as well as the time to train the technicians can be high. This results in a lot of support calls related specifically to the rebuild procedure for both new and experienced FSRs, causing downtime and damage to equipment.

PG\&E deployed a 3D interactive maintenance simulation (built by Heartwood) that allows the FSR to practice each action and step, as if they were tearing it down and rebuilding it in the real world. It includes choosing the appropriate tool at the relevant step and shows how this equipment works in the overall system. The simulation was designed to be portable, scalable and modular on iPads and PCs.

The editor allows users to design scenes, instances of the 3D landscape in Unity, and instantly observe the results of edits made. Unity also enables users to deploy their games to a myriad of platforms including TV, desktop, and mobile devices. Furthermore, Unity supports VR and augmented reality (AR), which makes virtual objects appear in the real world, and is used to create games for virtual reality headsets. Most games in Unity include features from the Unity asset store, which also allows users to access artwork, models, and scripts, programs that accomplish tasks in Unity. Finally, Unity allows users to share their work with others: Unity Teams allows for collaboration on projects while Unity Connect allows users to share their work with potential recruiters.

Labster is yet another platform that gives students access to a realistic lab experience that will let them perform experiments and practice their skills in a fun and risk-free learning environment.

\section{AR AND VR ADAPTABILITY}

Most of the available AR/VR tools enables students to learn by doing, using the various tools available with the technology. All features can be adapted to fit different class subjects and learning objectives. For Example, students can apply learning for social sciences, STEM and coding, literature, Languages, makerspace and arts.

\section{A. Social Sciences}

Infographics can be nice visual support for presentations in various formats like charts or tables, graphs and even timelines. Cospaces is a good development AR/VR tool that helps students to create an infographic, with a grid canvas to align objects. For example, charts can be created in CoSpaces using the various building blocks available in the library. You can use the cuboid building block in different heights to create a chart like a histogram that displays the distributions or the tube that can be cut as shown in Fig. 1. 


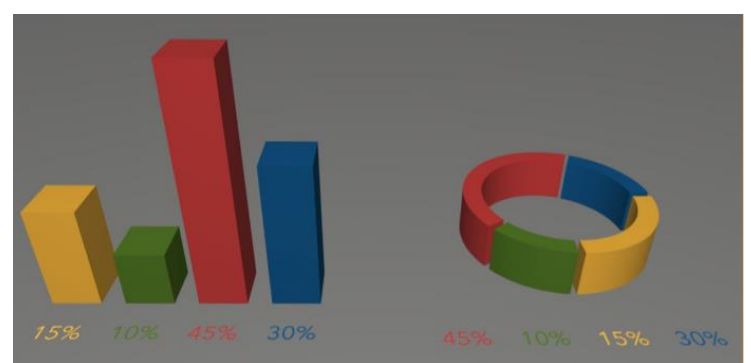

Fig. 1. Example of chart creations using cuboid tool in Cospaces.

\section{B. Architecture and Interior Design}

With CoSpaces creation tools, it's easy to build a house or apartment in 3D. Students can discover 3D creation and modelling through the construction and architecture of a virtual house or apartment. For example, floor plan image can be imported from the upload section and used as the basis on which to build house or apartment.
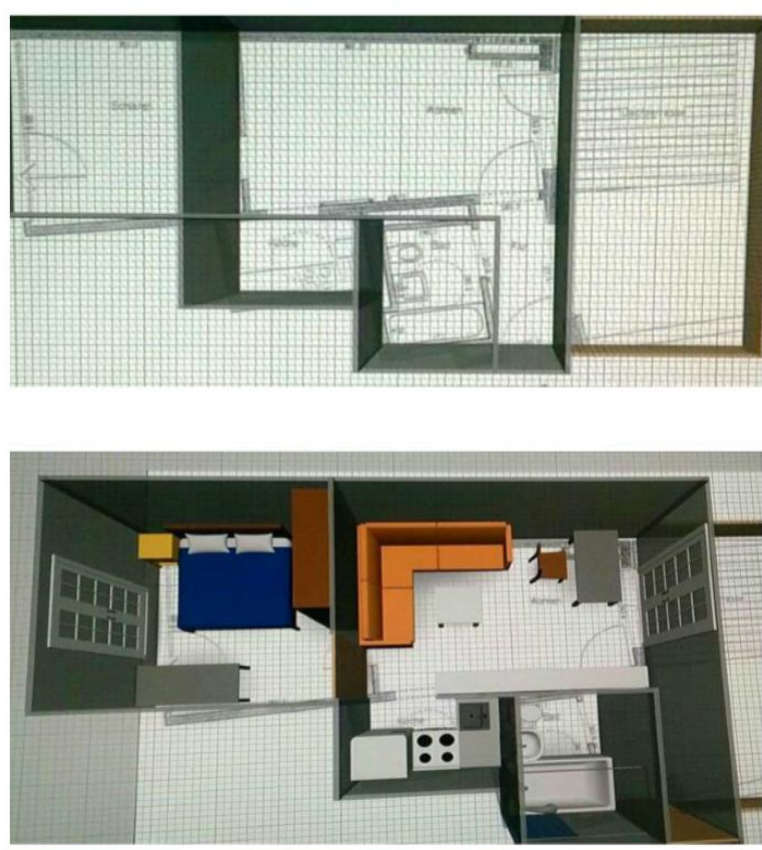

Fig. 2. Arcitecture and interior using design toolbox.

\section{STEM and Coding}

In addition to cuboid building tool, CoSpaces library contains a range of objects that students and teachers can use to recreate and simulate lab experiments. For example, Newton's Laws of motion by enabling the physics features of certain objects to allow for collisions, gravity and friction as shown in Fig. 2.

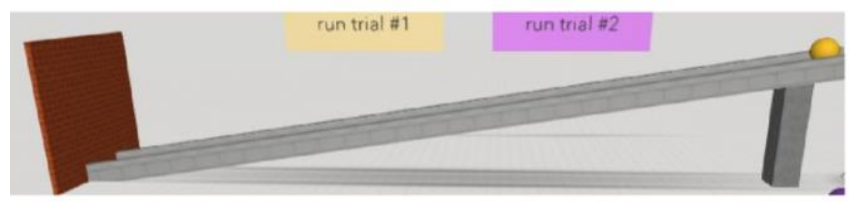

Fig. 3. Newton's laws using physics toolbox.

\section{EXPERIMENTAL VR DESIGN AND RESULTS}

Educators who teach in the experience age must utilize better methods to deliver the most effective learning experiences. One way to achieve this by integrate VR and its wide possibilities for learning, common misconception regarding virtual reality (VR) technology is that it is simply a gaming platform. Below is developed system, where VR is used to demonstrate an environment where he or she learns through interaction with the surroundings.

CoSpaces Edu offers proper solution for beginners who are getting started with coding, as well as scripting languages for more experienced coders. Students from first year of their studies can code their own VR environment using visual block-based and intuitive programming language, like scratch and easy for anyone to learn. Senior students can have fun coding scripts to add interactions and events. Every method contains a code sample and a demo project.

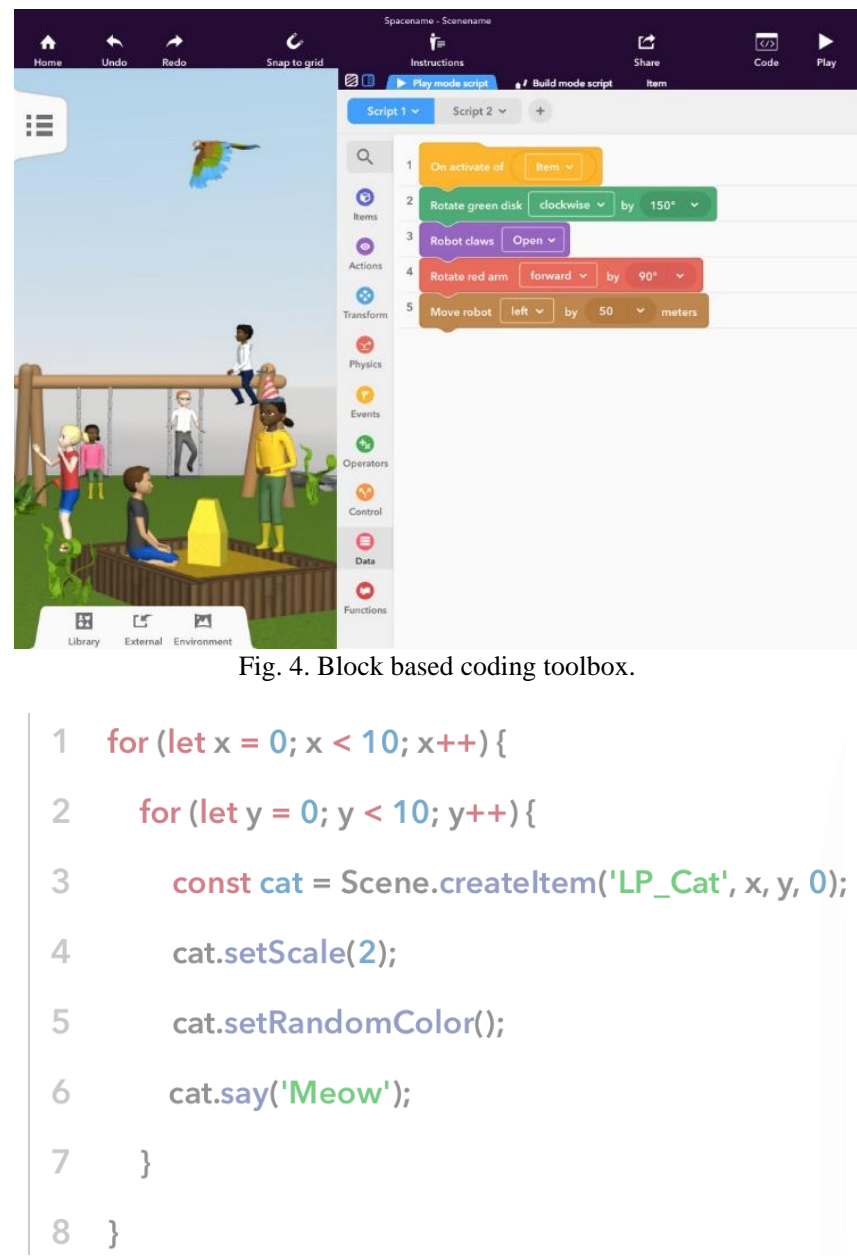

Fig. 5. Script coding toolbox.

\section{A. Water Cycle}

The water cycle is the process by which water undergoes changes in states through evaporation, condensation, and precipitation. Evaporation is the process through which water changes from a liquid into a gaseous substance. During condensation, gaseous particles of water come together and form liquid droplets. Finally, during precipitation, the liquid droplets fall back to earth in the form of rain, hail, or snow, or sleet. Some of these processes are depicted and developed in Cospaces.

During the design process students enabled physics toolbox in CoSpace. Physics toolbox activate real-world properties such as gravity and it makes the objects in the scene reacts as they would in real life. They can also define object mass, friction and bounciness, as well as more 
advanced properties like collision precision.

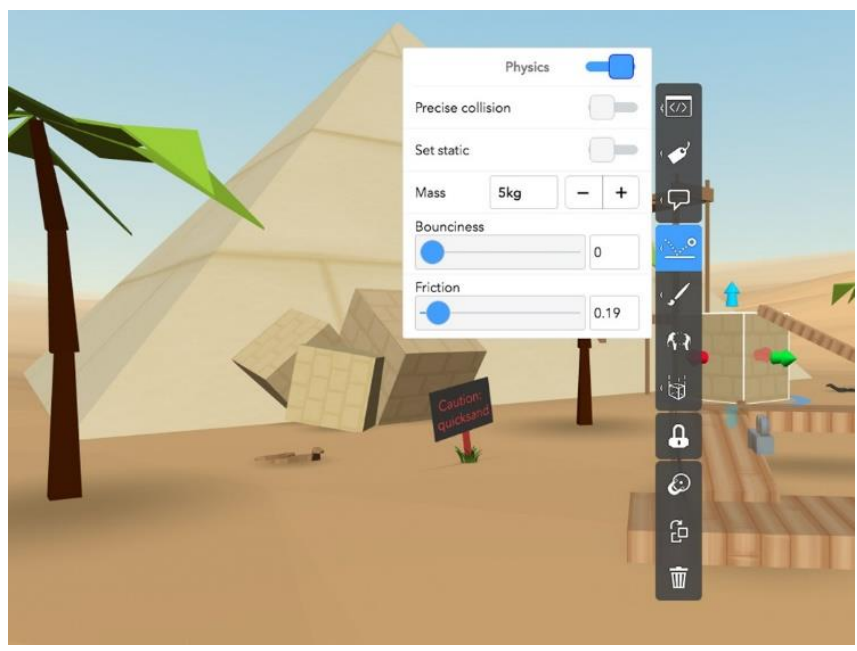

Fig. 6. Physics toolbox in cospaces.

First, a storyboard was created, defining the graphics and belongings required for each stage of the water cycle. Subsequently, the art resources and rail, which is a set path that the user would travel along, was created to transfer knowledge to the user through the stages of the water cycle. Additional art resources such as, more trees, water, and mountains as well as the sound of rain were developed into the environment to make it more interactive and increase user's interaction. The resources also included pictures for Burj Khalifa, UAE flag and the water cycle diagram. The viewer is given time to look around the scene, where they see trees, a mountain, a sunset, and water flowing all around them.

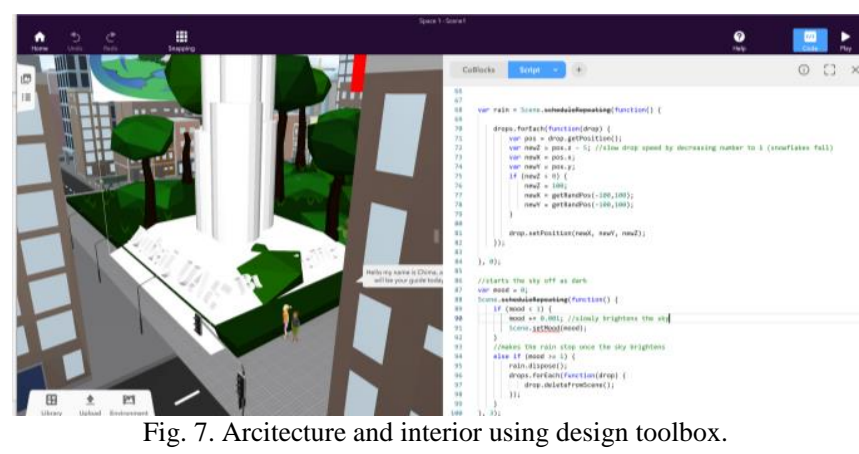

\section{B. Merge Cude Add-on}

The MERGE Cube is an add-on to the Cospaces platform. Where developers can easily create holograms in CoSpaces, as shown in Fig. 5. They will help students to interact and learn while holding their design on hands. Smartphones and tablets with iOS 9+ and Android 4.4+ can be used to view content on the MERGE Cube, using the CoSpaces Edu mobile app.

Energy by the sun increases the surface temperature of the Earth, which in turn heats up the water in rivers, lakes and oceans. When this happens, some of the water "evaporates" into the air, turning into a gas called vapour. Likewise, plants and trees lose water to the atmosphere through their leaves. This process is known as transpiration, as shown in Fig. 6. As water vapour increases and growths into the sky, it cools and turns back into a liquid, forming clouds. This process is called condensation. Clouds by turn moves around the globe, as shown in Fig. 7.

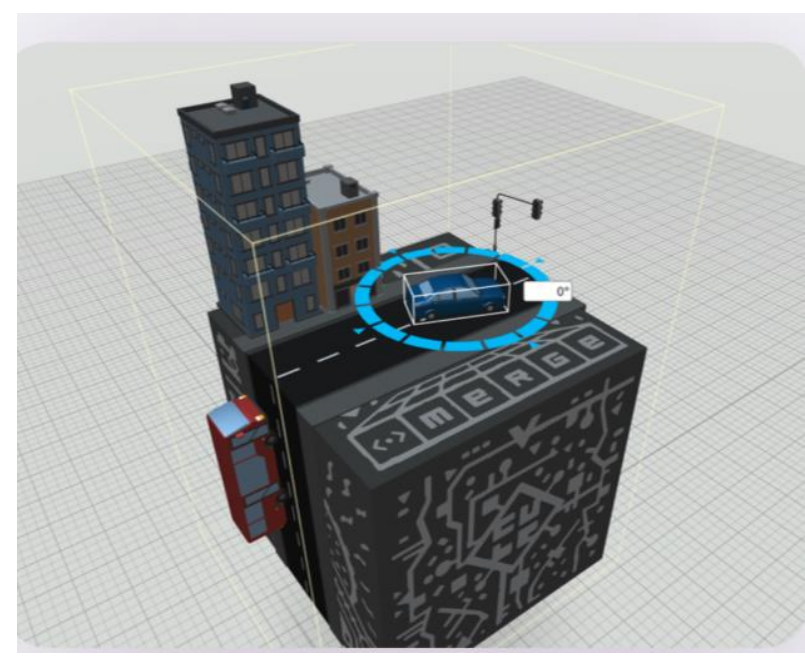

Fig. 8. MERGE cube add-on.

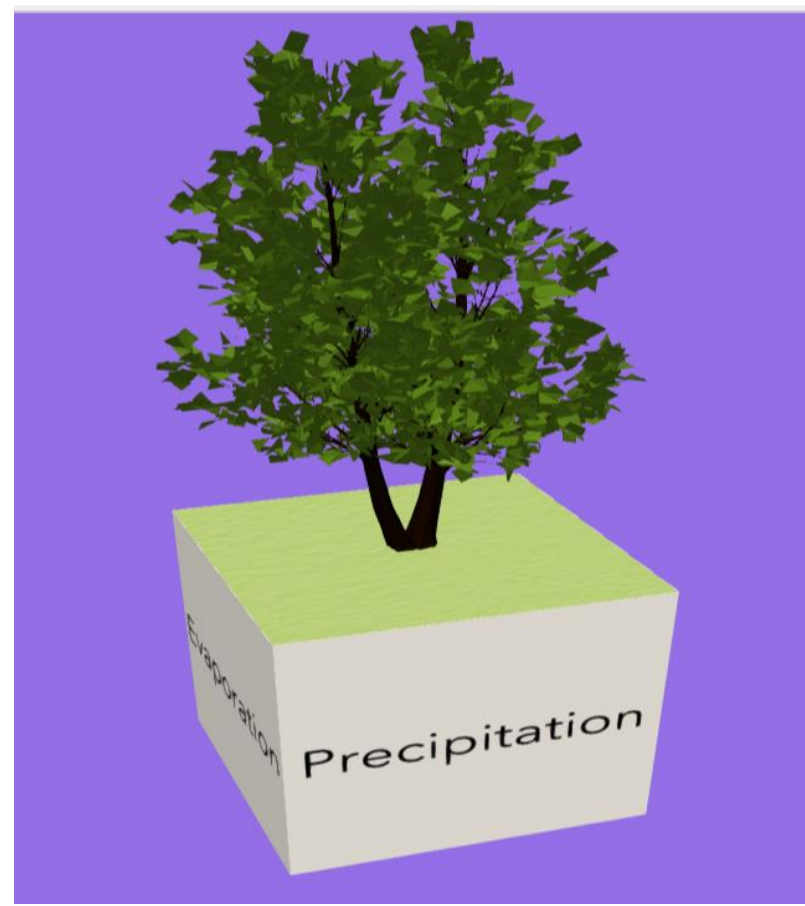

Fig. 9. Transpiration on MERGE cube.

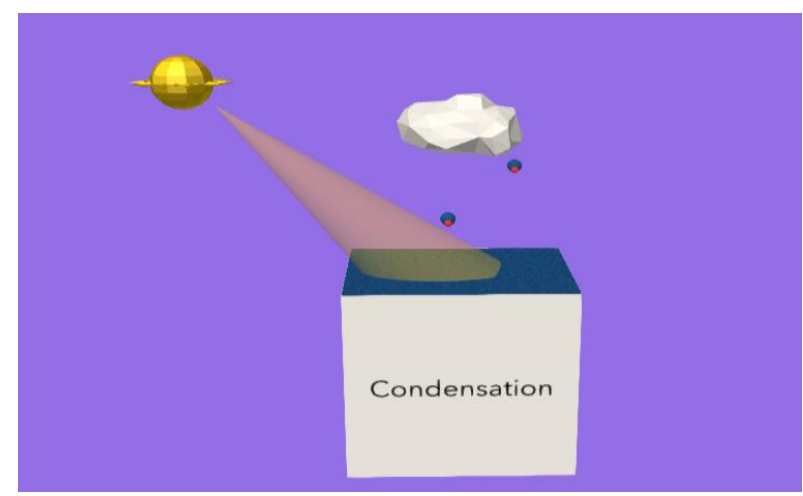

Fig. 10. Transpiration on MERGE cube.

\section{CONClusion}

Virtual reality can be used in education to create a more integrated environment. The paper presented how VR 
platform are being recently utilized in Automation, Architecture, Gas and oil industries an in education as well.

Water cycle has been developed using Cospaces VR platform to help young kids understand processes such as transpiration, vapour and condensation. Rather than using videos or charts that are typically used to explain the water cycle, using VR allows the user to feel more personally connected with their surroundings and helps them learn more effectively. As educators begin to look for more interactive methods to help their students learn, this research aimed to spread the benefits of VR technology to provide students with the proper amount of engagement and learning that they need to prepare for the future.

\section{FUTURE WORK}

The work presented here is a prototype for using Virtual reality in education. For education field it can help distance learners where VR interface can be used by students to be getting easier study of concepts and theories. Interior Design Students at universities can use VR 3D designs to enable higher level of understanding of a concept. It is also used for animations, and virtual experiences of the designs before real implementation. Beside the education services, Augmented and Virtual reality can be used for marketing. For example, exhibitions and event activations also make use of Augmented Reality for a more interactive way to display content. The work can be expanded on different directions and for different fields, such as real estate, tourism, education, coaching and training and medical industry. For tourism, VR can be used for hotel tours and attractions, travel destination, marketing of tourist packages and offers. For Medical, VR can help to minimize fear and anxiety from vaccinations, injections and blood draws for kids, teens and adults.

\section{CONFLICT OF INTEREST}

"The authors declare no conflict of interest".

\section{AUTHOR CONTRIBUTIONS}

Author 1: Al-Gindy revised/wrote the whole paper and conducted the literature review. Author 2 and 3: Chima and Ali developed the software using Cospaces and integrated the water cycle environments and steps. Authors 4 and 5: Munia and Amani, created the storyboard and enhanced the environment design and general look of the VR software. They have also added UAE themes to the design. Authors 2 to 5 conducted the similarity checking for whole paper. All authors had approved the final version.

\section{REFERENCES}

[1] J. Tokareva, "The difference between virtual reality, augmented reality and mixed reality," Forbes, 2018.

[2] M. Slater and M. V. Sanchez-Vives, "Enhancing our lives with immersive virtual reality," Frontiers in Robotics and AI, vol. 3, p. 74, 2016.

[3] M. Slater, "Place illusion and plausibility can lead to realistic behaviour in immersive virtual environments," Philosophical Transactions of the Royal Society B: Biological Sciences, vol. 364, no. 1535, pp. 3549-3557, 2009.

[4] N. Pellas et al., "Exploring the educational potential of three-dimensional multi-user virtual worlds for STEM education: A mixed-method systematic literature review," Education and Information Technologies, vol. 22, no. 5, pp. 2235-2279, 2017.

[5] J. Blascovich and J. Bailenson, Infinite Reality: Avatars, Eternal Life, New Worlds and the Dawn of the Virtual Revolution, New York: Harper Collins, 2011.

[6] B. Dalgarno and M. J. Lee, "What are the learning affordances of 3-D virtual environments?" British Journal of Educational Technology, vol. 41, no. 1, pp. 10-32, 2010.

[7] S. Freitas and G. Veletsianos, "Crossing boundaries: Learning and teaching in virtual worlds," British Journal of Educational Technology, vol. 41, no. 1, pp. 3-9, 2010.

[8] S. Freita and G. Veletsianos, British Journal of Educational Technology, 2010.

[9] M. Gardner et al., "Virtual laboratories for education in science, technology, and engineering: A review," Computers \& Education, vol. 95, pp. 309-327, 2016.

[10] G. Makransky et al., "Adding immersive virtual reality to a science lab simulation causes more presence but less learning," Learning and Instruction, 2017.

Copyright $\odot 2020$ by the authors. This is an open access article distributed under the Creative Commons Attribution License which permits unrestricted use, distribution, and reproduction in any medium, provided the original work is properly cited (CC BY 4.0).

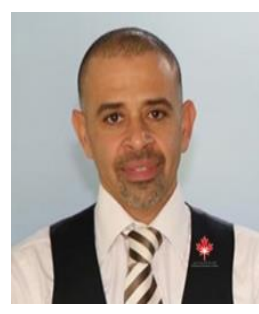

Ahmed Al-Gindy holds a Ph.D. in electrical and communication engineering from Faculty of Engineering and Informatics, University of Bradford, United Kingdom. He also obtained his M.Phil degree from Faculty of Engineering and Informatics University of Bradford United Kingdom, and a bachelor of engineering in electrical and computer engineering from Faculty of Engineering \& Technology, Maritime Academy for Science \& Technology, Alexandria Egypt.

Dr. Al-Gindy is a certified academic program assessor in the association of Arab Universities, Department of quality Assurance and quality enhancement in higher education institute. He is certified in Business process Management and Improvements, from George Washington University, USA. Dr. Al-Gindy has developed several curriculum and instruction Manuals in Engineering and Computing Technologies. His research interests cover design of various signal and image processing algorithms, RFID technologies and Artificial Intelligence. 